Nota científica

\title{
Primer registro de poblaciones silvestres de Cherax quadricarinatus (Decapoda: Parastacidae) en Sinaloa, México
}

\author{
First record of wild populations of Cherax quadricarinatus (Decapoda: Parastacidae) in Sinaloa, \\ Mexico
}

\author{
Edith Hilario Torres-Montoya ${ }^{\text {a }}$, Víctor Manuel Salomón-Soto a, Marcos Bucio-Pacheco ${ }^{\text {a }}$, \\ José Israel Torres-Avendaño ${ }^{a}$, Michelle López-Ruiz ${ }^{a}$, Sergio Sánchez-Gonzáles ${ }^{a}$ \\ e Hipólito Castillo-Ureta ${ }^{\text {b,* }}$

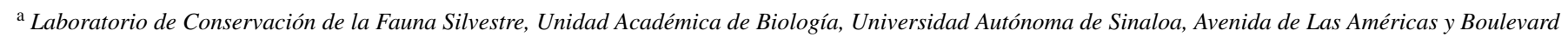 \\ Universitarios s/n, 80010, Culiacán, Sinaloa, México

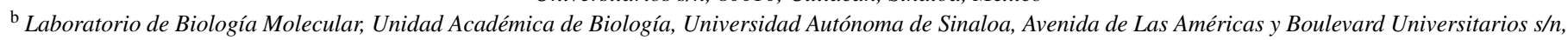 \\ 80010, Culiacán, Sinaloa, México
}

Recibido el 4 de agosto de 2015; aceptado el 21 de septiembre de 2015

Disponible en Internet el 9 de febrero de 2016

\begin{abstract}
Resumen
La langosta de quelas rojas australiana (Cherax quadricarinatus) fue introducida a México hace una década. Recientemente se han registrado poblaciones silvestres en distintas partes del país. Esta nota es el primer registro de C. quadricarinatus en medio silvestre en Sinaloa, México. Doce ejemplares adultos fueron capturados en distintos puntos del río Culiacán entre enero y marzo de 2015.

Derechos Reservados (C) 2015 Universidad Nacional Autónoma de México, Instituto de Biología. Este es un artículo de acceso abierto distribuido bajo los términos de la Licencia Creative Commons CC BY-NC-ND 4.0.
\end{abstract}

Palabras clave: Fauna nativa; Especie invasora; Cherax quadricarinatus; Río Culiacán

\begin{abstract}
The Australian red claw crayfish (Cherax quadricarinatus) was introduced into Mexico a decade ago. Recently wild populations have been reported in different parts of the country. The aim of this paper is to report the first record of C. quadricarinatus in the wild in Sinaloa, Mexico. Twelve adult specimens were collected in different parts of the Culiacan River between January and March 2015.

All Rights Reserved (C) 2015 Universidad Nacional Autónoma de México, Instituto de Biología. This is an open access item distributed under the Creative Commons CC License BY-NC-ND 4.0.
\end{abstract}

Keywords: Native fauna; Invasive species; Cherax quadricarinatus; Culiacan River

Cherax quadricarinatus (Von Martens, 1868) conocido comúnmente como langosta de quelas rojas es un decápodo de agua dulce perteneciente a la familia Parastacidae, es endémica del norte de Australia y sureste de Papúa Nueva Guinea (Lawrence y Jones, 2002). Se caracteriza por tener conducta

\footnotetext{
* Autor para correspondencia.

Correo electrónico: hipolito.cu@ hotmail.com (H. Castillo-Ureta).

La revisión por pares es responsabilidad de la Universidad Nacional Autónoma de México.
}

gregaria, es de rápido crecimiento y con alta plasticidad ecológica. Puede resistir variaciones de temperatura, $\mathrm{pH}$ y diferentes concentraciones de oxígeno disuelto (Karplus et al., 1998), por lo que es considerada una buena especie para el cultivo en condiciones semintensivas e intensivas, ya que, además, su dieta es omnívora (Edgerton, 2005; Jones, 1990; Lawrence y Jones, 2002).

Se ha visto que la producción de la langosta en estanques abiertos tiene mejores resultados que en sistemas de circulación cerrados (Rodríguez-Canto, Arredondo, Ponce-Palafox y Rouse, 2002). La esencia de la producción no tecnificada 


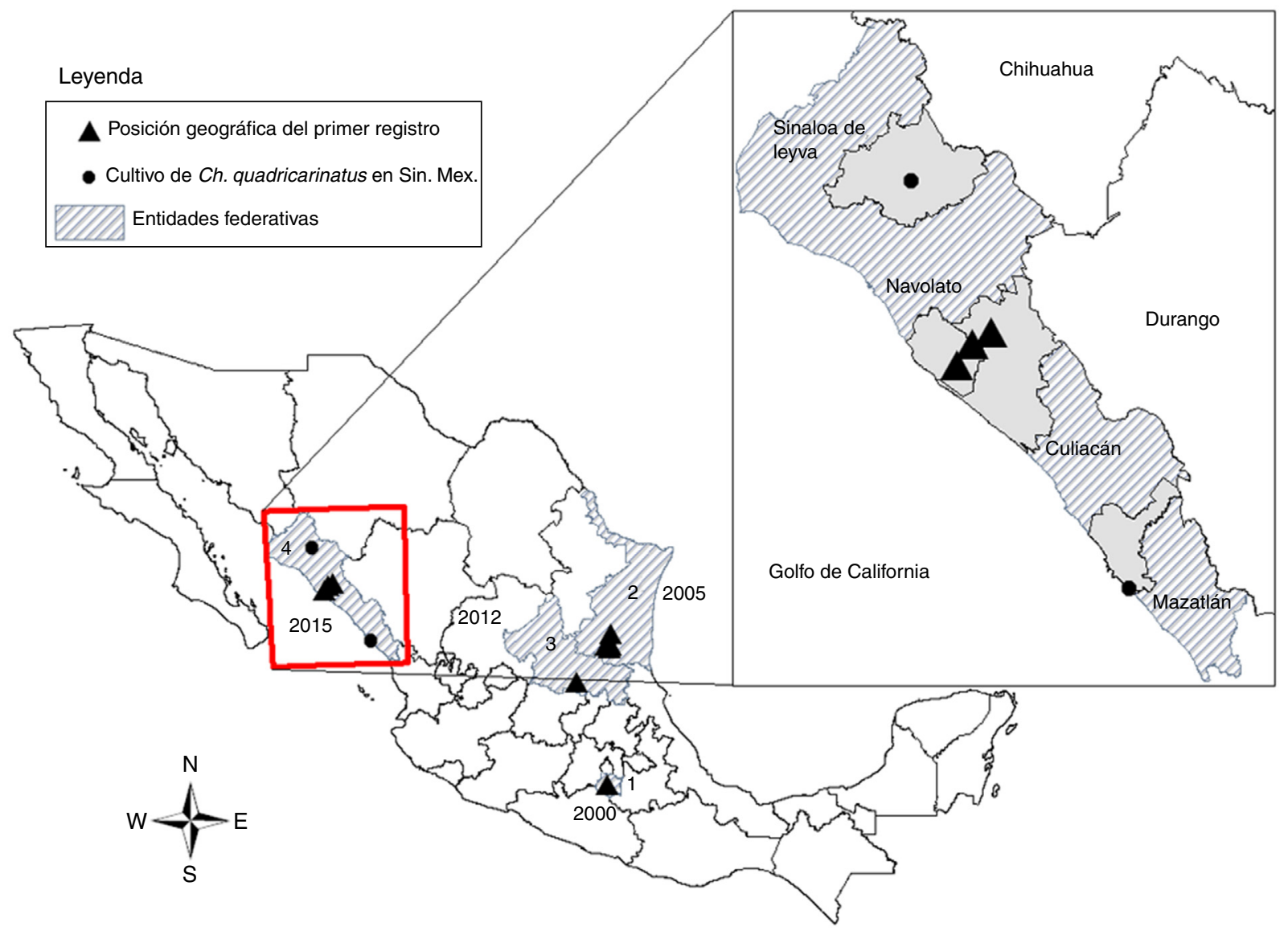

Figura 1. Primeros registros de poblaciones silvestres de C. quadricarinatus en México por año y entidad federativa: Morelos (1); Tamaulipas (2); San Luis Potosí (3) y Sinaloa (4).

implica, normalmente, que se hagan manejos descuidados durante los procesos de producción, lo que suele tener como consecuencia que algunos ejemplares se escapen al medio silvestre. Este mal uso de los sistemas de cultivo en estanques abiertos es la razón que se atribuye para que $C$. quadricarinatus haya invadido distintos efluentes (Bortolini, Álvarez y Rodríguez-Almaraz, 2007). En consecuencia y dado su amplio cultivo en distintas partes de Asia (Ahyong y Yeo, 2007), América (Bortolini et al., 2007), África (De Moor, 2002), Australia (Edgerton, 2005) y más recientemente en Israel (Snovsky y Galil, 2011), se han establecido diferentes poblaciones en distintos cuerpos de agua en los países donde se practica su cultivo.

En México, la langosta de quelas rojas fue introducida en 1995 con fines de investigación en la Ciudad de México y Yucatán; para el año 1998 ejemplares de esta especie fueron trasladados a Morelos, Baja California y Baja California Sur, Tamaulipas, Colima y Jalisco (Ponce-Palafox, ArredondoFigueroa y Romero, 1999). En la actualidad se sabe de la presencia de la especie en Aguascalientes, Chihuahua, Michoacán, Nuevo León, Puebla, Veracruz, Oaxaca, San Luis Potosí, Sinaloa y Veracruz (Álvarez, Bortolini, Villalobos y García, 2014). A más de una década de su introducción se registran poblaciones silvestres de la especie en cuerpos y afluentes de agua dulce en los estados de Morelos, Tamaulipas y San Luis Potosí (Álvarez et al., 2014; Bortolini et al., 2007). En Sinaloa se han mantenido individuos de la especie para fines de producción e investigación cerca de Mazatlán (Arzola-González et al., 2012) y Guasave (Álvarez et al., 2014), y no se sabía de su presencia en ecosistemas dulceacuícolas del estado.

Como parte de un estudio sobre la diversidad de crustáceos en el río Culiacán, se recolectaron 12 ejemplares adultos de $C$. quadricarinatus en presa derivadora, Culiacán, Sinaloa $\left(24^{\circ} 48^{\prime} 23.9^{\prime \prime} \mathrm{N} ; 1^{\circ} 7^{\circ} 24^{\prime} 50.4^{\prime \prime}\right.$ O); San Pedro (244 $47^{\prime} 06.1^{\prime \prime} \mathrm{N}$, $107^{\circ} 33^{\prime} 22.1^{\prime \prime}$ O) y Sataya $\left(24^{\circ} 37^{\prime} 48.6^{\prime \prime} \mathrm{N}, 107^{\circ} 39^{\prime} 41.1^{\prime \prime} \mathrm{O}\right)$, Navolato, Sinaloa (fig. 1). De los 12 individuos recolectados 5 fueron hembras y 7 machos (tabla 1), representando el primer registro en el estado de Sinaloa de dicha especie en condiciones silvestres, en donde algunos parámetros fisicoquímicos como temperatura y $\mathrm{pH}$ durante la inspección de campo oscilaron en un rango de 24 a $26^{\circ} \mathrm{C}$ y 7.2 a 8.1, respectivamente. En la figura 2

Tabla 1

Parámetros abióticos y datos de muestreo de C. quadricarinatus en el río Culiacán, en el estado de Sinaloa, México.

\begin{tabular}{lllll}
\hline Puntos de recolecta & $\begin{array}{l}\text { Núm. de } \\
\text { machos }\end{array}$ & $\begin{array}{l}\text { Núm.de } \\
\text { hembras }\end{array}$ & $\begin{array}{l}\text { Temperatura } \\
\left({ }^{\circ} \mathrm{C}\right)\end{array}$ & $\mathrm{pH}$ \\
\hline Culiacán, Sin. & 1 & 2 & 24 & 7.2 \\
San Pedro (Navolato, Sin.) & 2 & 0 & 26 & 7.7 \\
Sataya (Navolato, Sin.) & 4 & 3 & 26 & 8.1 \\
\hline
\end{tabular}




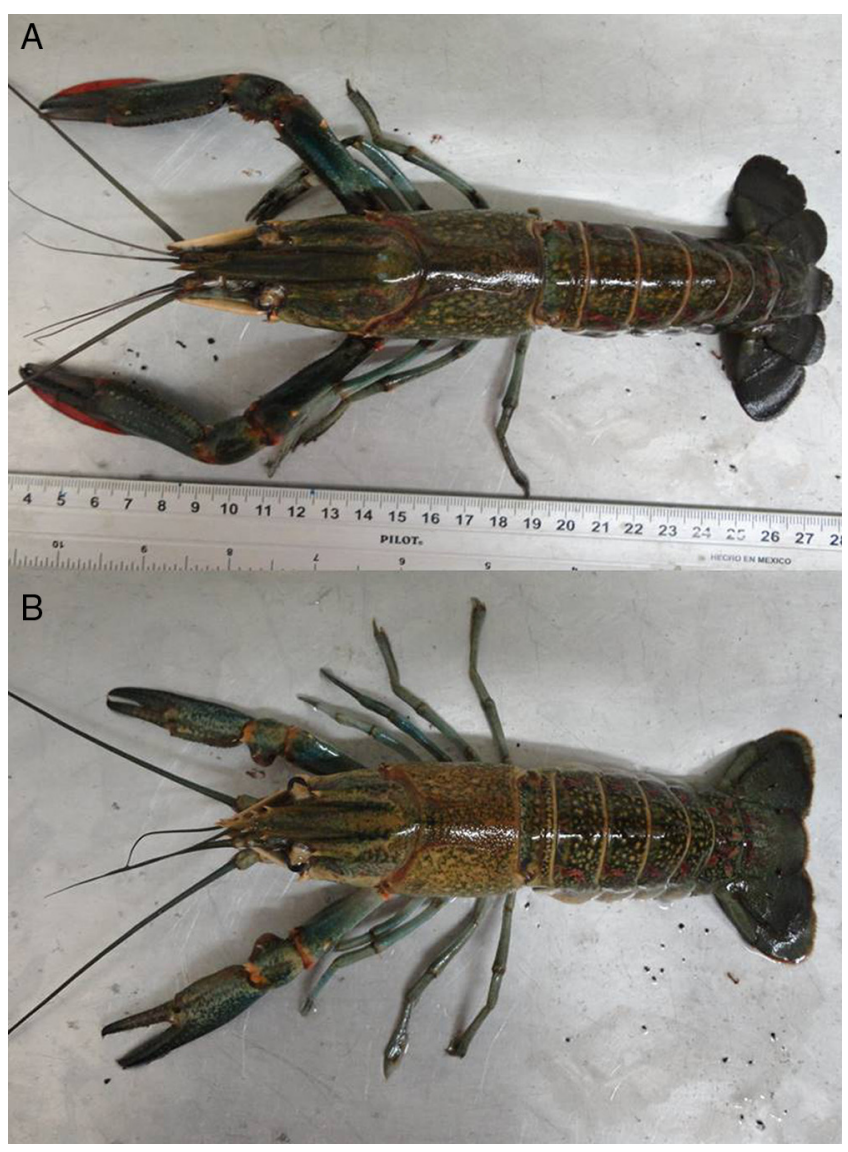

Figura 2. Macho (A) y hembra (B) de C. quadricarinatus. Una característica distintiva de los machos de la especie es la coloración roja en las quelas, ausente en hembras.

se muestran 2 ejemplares, uno de ellos con el parche rojo en las quelas, carácter distintivo de la especie (Snovsky y Galil, 2011).

Las especies invasoras acuáticas tienen efectos en los sitios donde han sido introducidas y se han establecido (Homans y Smith, 2013) y representan una de las amenazas más importantes para la biodiversidad de los ecosistemas acuáticos (Kalinowski, Muhlfeld, Guy y Cox, 2010), especialmente los dulceacuícolas que son altamente vulnerables al impacto de estas (Sala et al., 2000). A pesar de que se sabe que $C$. quadricarinatus es una especie invasora en México, se han realizado diferentes estudios para evaluar su potencial acuícola y promover su rentabilidad (Arzola-González et al., 2012; Hernández-Vergara, Rouse, Olvera-Novoa y Davis, 2003). Por lo anterior, es importante mencionar que se requiere una mayor vigilancia en el manejo de la langosta de quelas rojas por las autoridades competentes, ya que pudiera desplazar especies autóctonas como Macrobrachium americanum, Macrobrachium tenellum y Macrobrachium digueti y propiciar la difusión de nuevos parásitos entre la fauna nativa.
Agradecemos al Programa de Fomento y Apoyo a Proyectos de Investigación (PROFAPI 2013/040) de la Universidad Autónoma de Sinaloa y al Instituto de Apoyo a la Investigación e Innovación (INAPI) por otorgar facilidades para la realización del presente trabajo.

\section{Referencias}

Ahyong, S. T. y Yeo, D. C. J. (2007). Feral populations of the Australian red-claw crayfish (Cherax quadricarinatus Von Martens) in water supply catchments of Singapore. Biological Invasions, 9, 943-946.

Álvarez, F., Bortolini, J. L., Villalobos, J. L. y García, L. (2014). La presencia del acocil australiano Cherax quadricarinatus (Von Martens, 1868) en México. En A. M. Low-Pfeng, P. A. Quijón y E. Peters-Recagnos (Eds.), Especies invasoras acuáticas: casos de estudio en ecosistemas de México (pp. 603). México D.F.: Secretaría de Medio Ambiente y Recursos Naturales.

Arzola-González, J. F., de la Cruz, E. M., Verde-Osuna, L. I., García-Burgueño, E., Flores-Campaña, L. M. y Gutiérrez-Rubio, Y. (2012). Crecimiento, densidad y rentabilidad del cultivo de langosta de agua dulce (Decapoda: Cherax quadricarinatus) en Sinaloa, México. Ra Ximhai, 8, 17-26.

Bortolini, J., Álvarez, F. y Rodríguez-Almaraz, G. (2007). On the presence of the Australian redclaw crayfish, Cherax quadricarinatus, in Mexico. Biological Invasions, 9, 615-620.

De Moor, I. (2002). Potential impacts of alien freshwater crayfish in South Africa. African Journal of Aquatic Science, 27, 125-139.

Edgerton, B. F. (2005). Freshwater crayfish production for poverty alleviation. World Aquaculture Society, 36, 48-64.

Hernández-Vergara, M. P., Rouse, D. B., Olvera-Novoa, M. A. y Davis, D. A. (2003). Effects of dietary lipid level and source on growth and proximate composition of juvenile redclaw (Cherax quadricarinatus) reared under semi-intensive culture conditions. Aquaculture, 223, 107-115.

Homans, F. R. y Smith, D. J. (2013). Evaluating management options for aquatic invasive species: concepts and methods. Biological Invasions, 15, 7-16.

Jones, C. M. (1990). The biology and aquaculture potential of the tropical freshwater crayfish, Cherax quadricarinatus. Brisbane: Queensland Department of Primary Industries.

Kalinowski, S. T., Muhlfeld, C. C., Guy, C. S. y Cox, B. (2010). Founding population size of an aquatic invasive species. Conservation Genetics, 11, 2049-2053.

Karplus, I., Zoran, M., Milstein, A., Harpaz, S., Eran, Y., Joseph, D., et al. (1998). Culture of the Australian red-claw crayfish (Cherax quadricarinatus) in Israel III. Survival in earthen ponds under ambient winter temperatures. Aquaculture, 166, 259-267.

Lawrence, C. y Jones, C. (2002). Cherax. En D. M. Holdich (Ed.), Biology of freshwater crayfish (pp. 645-666). Oxford: Blackwell Science.

Ponce-Palafox, J. T., Arredondo-Figueroa, J. L. y Romero, X. (1999). Análisis del cultivo comercial de la langosta de agua dulce (Cherax quadricarinatus): y su posible impacto en América Latina. Contactos, 31, 54-61.

Rodríguez-Canto, A., Arredondo, J. L., Ponce-Palafox, J. y Rouse, D. B. (2002). Growth characteristics of the Australian redclaw crayfish, Cherax quadricarinatus, cultures in an indoor recirculating system. Journal of Applied Aquaculture, 12, 59-64.

Sala, O. E., Stuart-Chapin, F. I., Armesto, J. J., Berlow, E., Bloomfield, J., Dirzo, R., et al. (2000). Global biodiversity scenarios for the year 2100. Science, 287, 1770-1774.

Snovsky, G. y Galil, B. S. (2011). The Australian redclaw crayfish Cherax quadricarinatus (Von Martens, 1868) in the sea of Galilee, Israel. Aquatic Invasions, 6, 29-31. 